\title{
Civilisations
}

Revue internationale d'anthropologie et de sciences

humaines

52-1 | 2004

Transmission des savoirs et interactions culturelles

\section{L'hermétisme et la gnose : tradition et distorsion dans la transmission du savoir}

\section{Michèle Broze}

\section{OpenEdition \\ Journals}

Édition électronique

URL : http://journals.openedition.org/civilisations/739

DOI : 10.4000 /civilisations.739

ISSN : 2032-0442

\section{Éditeur}

Institut de sociologie de l'Université Libre de Bruxelles

\section{Édition imprimée}

Date de publication : 1 mai 2004

Pagination : $37-48$

ISSN : 0009-8140

Référence électronique

Michèle Broze, "L'hermétisme et la gnose : tradition et distorsion dans la transmission du savoir »,

Civilisations [En ligne], 52-1 | 2004, mis en ligne le 28 janvier 2009, consulté le 02 juin 2020. URL http://journals.openedition.org/civilisations/739 ; DOI : https://doi.org/10.4000/civilisations.739 


\section{L'hermétisme et la gnose : tradition et distorsion \\ dans la transmission du savoir}

\section{MICHĖLE BROZE}

Dans Les limites de l'interprétation, Umberto Eco entame une description de ce qu'il nomme un « modèle fort de l'hermétisme ». Il oppose le « rationalisme grec », représenté par Platon et Aristote, et qui construit le savoir sur la recherche des causes, à la pensée hermétique, qui anéantit l'ordre causal et « transforme le théâtre du monde en un phénomène linguistique et, parallèlement, retire au langage tout pouvoir communicatif ». Puisque tous les éléments du cosmos se répondent et renvoient sans cesse les uns aux autres (tout se tient), l'interprétation est infinie et infiniment en spirale. Le langage perd son pouvoir communicatif, parce qu'il déplace le récepteur continuellement d'un secret à un autre, et qu'il « ne peut y avoir de secret final. Le secret final de l'hermétisme est un secret vide ». Dans ce cas, dialogue et raisonnement sont inutiles, car la connaissance viendra d'une illumination extérieure et fulgurante ${ }^{1}$.

C'est un modèle fort, dit Eco, et peut-être, à mon sens, trop contrasté. En effet, la transmission et la construction du savoir sur le monde ne sont pas chez Platon exclusivement de l'ordre d'une causalité du type post hoc ergo propter hoc. La figure du politique, par exemple, émerge dans une ambiguïté temporelle et spatiale, comme le fait la fameuse cité platonicienne. Tout en étant un modèle passé, elle se construit dans le futur, et si elle est présente ici et maintenant, c'est « comme un dieu parmi les hommes ». Rappelons encore que selon Platon, le soleil est fils du Bien, tout en étant inengendré. Couloubaritsis a démontré comment ces contradictions se résolvaient si l'on conçoit qu'elles témoignent d'une pratique du mythe et dans le cas du soleil, fils du Bien, d'une exploitation du schème de la parenté 2 .

L'analyse d'Eco suppose que c'est la vision du monde de l'hermétisme (la sympathie universelle) qui provoque ce jeu infini de reflets dans les miroirs du monde et cette pratique du raisonnement circulaire qui pose l'effet comme la cause.

Comme déviante du rationalisme gréco-latin, Umberto Eco cite dans le même contexte historique la pensée gnostique, pour qui le monde est l'œuvre d'un démiurge mauvais et déficient. L'accès au savoir véridique, qui se fait par des voies initiatiques

1. Eco $1992: 51-63$.

2. Couloubaritsis 1982 ; Broze 1995. 
comme dans l'hermétisme, suppose également un agent extérieur qui aidera à cerner dans les textes de la tradition la vérité dissimulée par l'ignorance de ceux qui les ont livrés.

Cela implique-t-il pour autant que le monde s'interprète à l'infini, ou que la polysémie du langage hermétique ou gnostique n'arrive qu'à un vide de sens? J'essaierai de montrer que la manière dont les auteurs de ces deux courants de pensée élaborent leurs textes a sa logique propre et que les curieux effets de spatialité et de temporalité qu'ils produisent ont un sens et une fonction qui se perçoivent si l'on examine les documents sous l'angle de la pragmatique : il s'agit bien de mettre en œuvre des processus de validation qui donnent au contenu et au processus d'accès au savoir une valeur de vérité.

L'enseignement hermétique est un enseignement initiatique, qui se transmet de maître à disciple, situation souvent thématisée par un lien familial de type père-fils. Cette forme dialoguée sert de cadre narratif ${ }^{3}$ au contenu de la révélation : dans l'Asclépius, par exemple, Hermès Trismégiste propose à Asclépius d'appeler son fils Tat, et Asclépius suggère d'admettre aussi Hammon à l'entretien, qui se déroule dans un sanctuaire. Dans le traité XVI, le cadre narratif est celui d'une lettre envoyée au roi Ammon, lettre écrite fictionnellement en égyptien ${ }^{4}$, et dont le roi doit empêcher la traduction en grec, langue inapte à dire le vrai, car elle ne contient pas l'energeia, la performativité, mais se réduit à la reconstruction de la chaîne causale (on voit que les auteurs de l'hermétisme donneraient raison à Eco).

L'utilisation du modèle parental n'est toutefois pas le simple reflet d'une tradition dans la transmission des savoir-faire, comme le pense Festugière. Comme l'a montré M. Kasajima pour le traité XIII du Corpus hermétique, le statut de la relation père-fils peut être exploité comme un élément moteur dans la narration : la filiation paternelle devient le lieu d'un jeu sur l'identité du père, qui se tisse peu à peu au fil d'un dialogue. La structure dialoguée, loin d'être simplement didactique, est indispensable à cette construction ambiguë de la figure du père ${ }^{5}$.

Ce traité est intitulé D'Hermès Trismégiste à son fils Tat. Discours de révélation (apocryphe) sur la montagne, au sujet de la régénération et de la règle du silence. Ce discours est un discours de salut, il est même la condition indispensable au salut. Tat, le fils, énumère les conditions à remplir pour l'obtenir : il faut se faire suppliant lors de la traversée de la montagne (ou du désert ${ }^{6}$ ), dialoguer avec le maître après avoir formulé explicitement sa demande d'apprendre un tel discours parce que c'est le seul qu'on ignore encore (il faut donc avoir reçu l'instruction préalable complètement), et,

3. Festugière, dans l'immense travail qu'il consacra à l'hermétisme (Festugière 1944-1954), classifie ces cadres narratifs, qu'il interprète lui-même comme des fictions littéraires, dont la structure est d'origine égyptienne (et c'est d'ailleurs un des rares éléments dont il accepte de situer l'origine dans la vallée du Nil). Pour lui, c'est le mode de transmission des savoirs et des techniques de père en fils qui inspira ce type de fiction, et sa présence constante dans les textes d'inspiration hermétique témoigne d'une mode littéraire à laquelle les auteurs doivent sacrifier.

4. Le texte fut bien évidemment écrit en grec. Sur le statut de cette fiction et sur le brouillage temporel qu'elle implique, voir Broze 1997.

5. Kasajima 2001.

6. Sur la montagne désignant les plateaux désertiques de part et d'autre de la vallée du Nil, Fowden 1986 : 40 . 
dernière condition posée par Trismégiste, il faut être prêt à se rendre étranger au monde. Tat déclare immédiatement remplir la dernière condition, il demande à recevoir le discours de la régénération et formule son ignorance : il ne sait de quelle matrice l'homme a été engendré, ni de quelle semence.

Instruit sans difficulté sur la matrice (la sagesse qui s'intellige dans le silence) et la semence d'où l'homme fut engendré (le vrai bien), il témoigne de son embarras au sujet de celui qui ensemence (le père, le principe) et de l'engendré (le fils/l'homme). Si le père est le vouloir de dieu, l'engendré est autre, dieu fils de dieu, le tout dans le tout, constitué à partir de toutes les puissances. "Tu me dis une énigme, père, et ne parles pas comme un père à un fils » : Tat reproche à Hermès de parler par énigme, comme il le faisait auparavant ${ }^{7}$. Ce mode d'enseignement ne répond pas au lien familial qui unit les deux interlocuteurs. A quoi le père répond qu'à cette question ne correspond pas d'enseignement (une structure traditionnelle père/fils), mais que la connaissance de ce genre (Tò yévos) demande une anamnèse donnée par le dieu.

La logique du dialogue suggère que Trismégiste est réticent à parler et comme pour le provoquer, Tat propose une parole de réponse ou de subsitution correcte

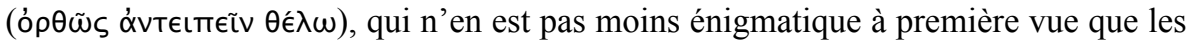

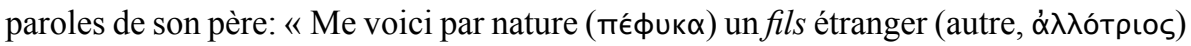
à la race paternelle. Ne te montre pas jaloux de ton savoir, père. Je suis un fils légitime. Explique-moi le mode de la régénération ». C'est comme si Tat explorait ici, pour déclencher l'initiation, les différentes modalités de la paternité, une paternité réelle dans la fiction, et spirituelle dans le processus décrit par la fiction, mais comme nous le verrons, une paternité divine qui le fera identique à son père.

La contre-réponse de Tat est bien correcte, car elle amène Trismégiste à faire voir sa propre régénération et à la faire vivre à son fils. Peut-être lui manquait-il de se faire suppliant, et le $\mu$ ทे $\phi \theta$ óveı lui permet de remplir cette condition. De même, pour être prêt à se rendre étranger au monde faut-il se déclarer étranger à la race paternelle, c'est à dire dépasser la filiation humaine et ne pas avoir encore accès à la filiation divine : on constate qu'à ce moment, toutes les conditions posées par Trismégiste au départ sont remplies, mais non sans ambiguïté au sujet de la race paternelle.

Et en réalité, comme Trismégiste l'avait précisé, le mode de transmission n'est pas celui de l'enseignement, mais bien celui de la réminiscence, non d'un contenu de savoir, mais d'une activité qui mène au savoir. Interrogé par Tat sur celui qui dans la ré-

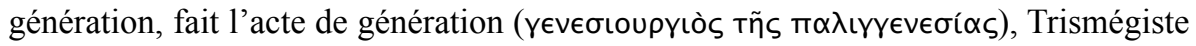
répond : "Le fils de dieu, l'homme un, par la volonté de dieu ». On s'en souvient, la volonté de dieu est « celui qui ensemence », et l'engendré est « autre, dieu fils de dieu ». On constate ici que l'opérateur de la régénération est également celui qui la vit. Père et fils de l'histoire (Hermès Trismégiste et Tat) deviennent dans ce processus issus d'un même père : " ignores-tu que te voici par nature (пє́ фuк $\alpha \varsigma$ ) dieu et fils de l'un, ce que je suis aussi? ». On ne peut manquer d'observer le parallélisme entre fils

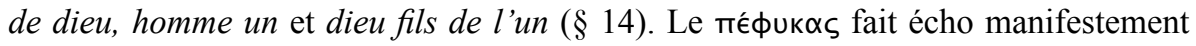
au те́фик $\alpha$ de Tat : ici, Tat n'est plus étranger à la race paternelle (celle de son père Trismégiste, qui est fils du dieu et celle de leur père commun). La généalogie est brouillée, le père et le fils devenant en quelque sorte frères.

7. Tout au début du texte, Tat signale que dans les entretiens précédents, Hermès parlait par énigme, sans dévoiler. 
Cette révélation a lieu quand, par le jeu de ce que Kasajima appelle plaisamment la fresque animée des puissances, la décade des puissances a vaincu la dodécade des punitions, sous les yeux et à l'appel d'Hermès et de Tat. Ces dix puissances, qui contiennent l'hénade et que l'hénade contient, composent le nouveau corps de Tat, qui est ainsi devenu l'engendré tel qu'il est défini au début (autre, dieu fils de dieu, le tout dans le tout, constitué à partir de toutes les puissances).

Ceci explique comment Trismégiste peut « chanter le seigneur de la création, le tout et l'un, par les puissances qui sont en lui-même », et comme l'ajoute Tat, " qui sont en tout ». L'hymne de Trismégiste clarifie le processus : s'adressant lui aussi au père, il le nomme « père, activité des puissances, et dieu, puissances de mes activités », ajoutant que ces puissances « accomplissent ta volonté, ton vouloir vient de toi et revient à toi » et que « C'est ton logos qui te chante à travers moi » $(\S 18)$ et « De ton logos, l'Intellect est le pasteur » $\left(\$ 19^{8}\right)$. Le lien entre logos et intellect renvoie bien ici l'activité démiurgique du père dont ils sont l'instrument, et c'est au démiurge que Trismégiste adresse ici son invocation $\delta \eta \mu$ เoupүé. Ceci suffirait à montrer que la régénération permet à l'engendré d'exercer en actes les puissances du démiurge qui sont en lui, de s'assimiler à lui par les puissances et les activités.

Tat le confirme, continuant le jeu généalogique qu'il avait commencé à pertuber au début. Au début de sa propre eulogie, il répond à Trismégiste : « Je dis ce que je con-

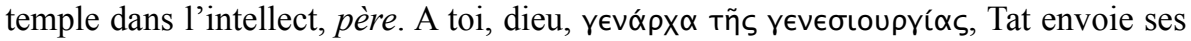
sacrifices en paroles. Dieu, tu es père, tu es seigneur tu es intellect, reçois de moi les sacrifices en paroles que tu veux. Car tout s'accomplit selon ton vouloir » (21). Ainsi, le dieu père est principe générateur de l'œuvre génératrice. On se souvient que celui

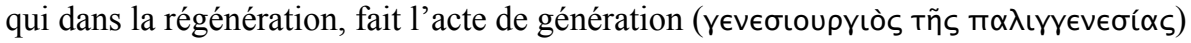
n'est autre que l'engendré issu lui-même de la régénération. En puissances et en activités, le dieu un et le fils de l'un s'assimilent. Père et fils de la fiction s'identifient aussi par la connaissance qu'ils ont d'eux-mêmes et de leur père commun, qui est le principe de leur activité (22).

On se demanderait peut-être avec irritation : à la fin, qui est père, qui est fils? Tourne-t-on sans fin dans une sémiosis illimitée. Si le fils est la cause de la naissance, n'a-t-on pas un de ces exemples de perversion de la rationalité grecque qu'évoquait Eco? Le père se déplaçant sans cesse, arrive-t-on à un vide de père? La polysémie du père entraîne-t-elle la perte du pouvoir communicatif du langage?

On notera d'abord que dans la famille hermétique, la généalogie n'est pas dépourvue d'ambivalence. Le statut de Trismégiste n'est pas facile à cerner : l'intervention du Nous divin associé au Logos divin fait de lui un dieu et fait de lui l'homme un, comme nous l'avons vu pour Tat, par l'intervention de Trismégiste. Ceci donnerait à penser que d'humains, ils deviennent divins. Pourtant, Trismégiste connaît son ancêtre, sa lignée véritable : son ancêtre est le dieu égyptien enterré à Hermopolis (le dieu Thot, maître et dispensateur du savoir divin par le biais de l'écriture hiéroglyphique, qualifiée de parole divine). Ceci implique qu'il y a en lui d'emblée du divin. Cette dualité est confirmée par le Poimandrès : l'intellect et le logos qui sont en lui sont divins, et c'est ce qu'il doit reconnaître. Pour lui comme pour Tat, l'initiation se produit par une reconnaissance du père, qui correspond à une reconnaissance de soi-même :

8. Ceci renvoie au traité $\mathrm{I}$, le Poimandrès, où Hermès Trismégiste reçoit du Nous une initiation qui le fait dieu et instructeur. 
« connais-toi toi-même et tu connaîtras dieu » se décline de manières multiples : ici, c'est « reconnais ton père et tu te reconnaîtras toi-même, et vice-versa ».

Ce processus de connaissance/reconnaissance est attesté dans quelques textes, mis en évidence déjà par Puech ${ }^{9}$. J'en ai parlé ailleurs et ne ferai ici que résumer ${ }^{10}$. Sous la houlette du dieu égyptien Thot, le savoir se dispense et se construit avec immédiateté, par le traçage des hiéroglyphes, qui font passer dans le visible le discours inaudible du dieu solaire, retiré loin des hommes. Ce modèle qui valide tout discours hiéroglyphique comme vrai ne fonctionne plus quand le grec se substitue à l'égyptien pour transmettre la révélation du savoir caché dans l'invisible : on se souvient que d'après le traité XVI du corpus (et c'est un topos), le grec ne sert qu'à produire des raisonnements qui ne contiennent pas l'energeia des choses. L'hermétisme, qui s'écrit en grec, opère une distorsion qui lui permet de valider autrement son contenu : il fragmente dans une succession généalogique la révélation et la place dans une absence de temporalité qui la rend permanente et universelle. Permanente dans le sens où elle ressurgit dans chaque grande période de l'histoire, et universelle dans la mesure où elle s'opère dans différents grands lieux qui symbolisent le savoir, comme l'Egypte, la Mésopotamie, l'Inde, et plus tard le monde arabe ${ }^{11}$.

Puisque le hiéroglyphe n'est plus moyen de communication entre divin et humain, la révélation s'actualise selon la succession des générations. Mais le révélateur est permanent. En effet, si Hermès est trois fois très grand (Trismégiste), il est aussi triple. On sait que l'épithète « trois fois très grand » est égyptienne, même si Thot est aussi « cinq fois très grand ». Le trois s'est imposé, et il faut constater dans la tradition égyptienne, Thot est « premier des trois », ou plus tard « un des trois $»^{12}$. La tradition a donc retenu le trois comme chiffre associé à l'Hermès égyptien, et l'empereur Julien y voit la trace de la supériorité de l'hermétisme sur le christianisme, puisque le Christ n'a qu'une double naissance, alors qu'Hermès s'incarne trois fois. De ceci, il apparaît que la succession des trois générations voit la génération d'un même Hermès. Les sources précisent en effet qu'Hermès a séjourné en Egypte trois fois et s'est reconnu

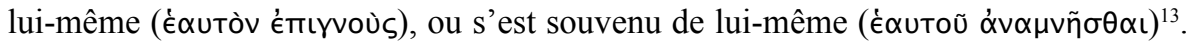
C'est le programme qu'Hermès propose ici à Tat, quand ce dernier lui reproche de ne pas parler comme un père à son fils : «Ce révos, enfant, ne s’enseigne pas, mais est le résultat d'une anamnèse opérée par le dieu quand il le veut » (úrò toũ $\theta \in$ ũ $\alpha \vee \alpha \mu \mu \nu \eta ́ \sigma \kappa \in T \alpha \iota)$. Nock pense à tort qu'il ne s'agit pas d'une réminiscence de soimême, comme dans les textes cités plus haut ${ }^{14}$. C'est pourtant bien la reconnaissance

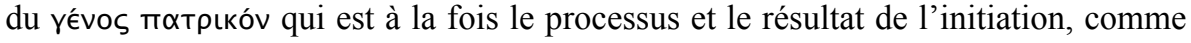

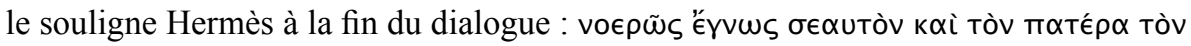
$\dot{n} \mu \in T$ té pov. Se connaître soi-même, c'est reconnaître son père et démiurge en intégrant

9. Puech 1978, vol. $1: 117-118$.

10. Broze 1997.

11. Mahé 1978 et Lory 1988.

12. Premier des trois notamment dans les Textes des Sarcophages, un des trois dans le temple romain d'Esna.

13. Ces textes, l'extrait de Julien et les autres, qui sont des scolies d'Hermias à Platon, sont cités dans Puech 1978.

14. Festugière-Nock 1983 (1954) : 149. 
en soi ses puissances et ses activités, en usant activement du nous et du logos divins.

L'intérêt du traité est de jouer sur la polysémie du lien père-fils, comme modèle de transmission des savoirs, et sur le statut de la légitimité du fils. L'auteur use ici de la tradition égyptienne à la fois dans ses pratiques sociales et dans ses pratiques du mythe. Le premier point est évident, le second mérite un développement : dans les fondements du mythe de la succession royale, le fils, Horus, hérite parce qu'il est fils légitime de son père Osiris, mais cette légitimité doit être confirmée par le père décédé. Il faut encore souligner que dans la théologie égyptienne, le fils naissant est aussi le père renaissant, posant ce même jeu de perturbation généalogique que dans la pensée hermétique ${ }^{15}$. La philosophie grecque, toutefois, n'est pas absente de l'utilisation, dans la dynamique du dialogue, du lien père-fils pour identifier/reconnaître le fils. Dans une belle étude, Marie-Laurence Desclos a mis en lumière un jeu platonicien sur la mention de la filiation ${ }^{16}$ : que de l'examen du fils encore anonyme, on remonte à la reconnaissance du père (causalité inversée), puis à l'identification du fils par son nom, ou plus simplement que du nom du père, on passe à celui du fils, la recherche de l'identité du fils par celle du père institue dans le dialogue platonicien une temporalité philosophique qui emprunte au temps civique pour le détourner de sa signification. Notons que Proclus lui-même commente la mention de la filiation paternelle dans les dialogues platoniciens comme un appel à se tourner vers le père divin pour se connaître lui-même. C'est la raison pour laquelle, dit-il, Socrate interpelle Alcibiade en le nommant d'abord « fils de Clinias $»^{17}$. Dans notre traité, il ne s'agit pas de créer une temporalité philosophique (une historialité), mais d'englober tous les temps, ce que fait Tat une fois son nouveau corps constitué.

On conviendra en fin d'analyse que la structure dialoguée, jugée inutile dans le modèle d'enseignement hermétique par Eco, est dans le traité XIII non un simple cadre littéraire à la révélation du secret, mais une méthode qui permet à l'initié de découvrir, en expérimentant la polysémie des mots, son identité avec le père de la fiction et avec le père et démiurge, et à l'auteur d'opposer et d'unir les différentes significations pour construire dynamiquement un sens.

Les cadres narratifs des textes gnostiques ne sont plus gratuits. Si le monde, créé par un démiurge déficient, est une erreur, l'initié apprendra à trouver dans des textes faux, les traces de la vérité. L'apprentissage de cette méthode exégétique se fait lors d'une révélation, sous l'autorité du Sauveur lui-même, qui enseigne à trouver le vrai sens. Un sens qui, bien entendu, sera écarté résolument par les Pères :

Rejetant la vérité, certains introduisent des discours mensongers et " des généalogies sans fin, plus propres à susciter des questions", comme le dit l'Apôtre, " qu'à bâtir l'édifice de Dieu fondé sur la foi » (1Tim 1, 4). Par une vraisemblance frauduleusement agencée, ils séduisent l'esprit des ignorants et les réduisent à leur merci, falsifiant les paroles du Seigneur et se faisant les mauvais interprètes de ce qui a été bien exprimé. Ils causent ainsi la ruine d'un grand nombre, en les détournant, sous prétexte de "gnose ", de Celui qui a constitué et ordonné cet univers : comme s'ils pouvaient montrer quelque chose de plus élevé et de plus grand que le Dieu qui

15. Sur le processus de reconnaissance paternelle dans le mythe osirien, voir Broze 1996.

16. Desclos 2000.

17. Proclus, Sur le premier Alcibiade de Platon, 24-25 (édition A.P. Seconds). 
a fait le ciel, la terre et tout ce qu'ils renferment! De façon spécieuse, par l'art des discours, ils attirent d'abord les simples à la manie des recherches; après quoi, sans plus se soucier de la vraisemblance, ils perdent ces malheureux, en inculquant des pensées blasphématoires et impies à l'endroit de leur créateur à des gens incapables de discerner le faux du vrai.

La préface du Contre les hérésies de l'évêque de Lyon, Irénée, indique clairement que l'hérétique, pour lui, est l'adepte d'une « fausse gnose ». Il faut d'emblée préciser que les courants de pensée critiqués et dénigrés par les Pères de l'Eglise, et qu'ils qualifient d'hérétiques, sont pour ainsi dire mis dans le même sac et dénommés " gnostiques ». Il convient encore de souligner que le terme " gnostique », sans doute à cause de la composante ésotérique du gnosticisme, finit par devenir pratiquement synonyme d'ésotérique, ce qui, pour les utilisations contemporaines, notamment chez R. Ruyer et chez $\mathrm{J}$. Charon, amène $\mathrm{M}$. Tardieu à parler de « charabia prétendu gnostique $\rangle^{18}$. On retiendra également l'utilisation littéraire du terme, bien illustrée par Lawrence Durrel dans le Quartet d'Alexandrie, où la ville elle-même et ce qui fait sa magie sont décrits en terme de gnose ; c'est un ésotérisme initiatique qui est ainsi désigné, avec référence à la pratique de la kabbale et au corpus hermétique.

Nous nous limiterons ici à ce que l'on peut appeler le gnosticisme historique : un courant de pensée chrétien qui, à partir du premier siècle de notre ère, développe une interprétation des textes sacrés fondée sur une révélation qui en garantit l'authenticité $^{19}$. Cette gnose, cette connaissance du vrai sens des Ecritures, donne à qui la reçoit le salut dans notre monde et notre temps. Dans la pensée gnostique, nous le verrons, le thème de la connaissance initiatique est central, tant dans l'acquisition de l'état d' « élu », de « parfait », que dans la structure des mythes. Le gnosticisme est donc structurellement ésotérique, et la révélation initiatique sert de schème unificateur entre l'invisible et le visible.

Précisons d'abord que si la Loi judaïque constitue bien pour les gnostiques une référence, le sens correct à donner au texte a été perdu. L'histoire de cette perte et l'interprétation exacte du texte passe par une relecture de l'Ancien Testament, souvent soutenue par une relecture gnostique du Nouveau Testament.

C'est le cas de l'Apocryphon de Jean, ou Livre des secrets de Jean, récit des secrets révélés par Jésus après sa mort à l'apôtre Jean, lors d'une apparition spectaculaire. Une des versions conservées précise : enseignement du Sauveur et révélation des mystères dès l'origine cachés dans le silence, toutes choses qu'il a enseignées à Jean, son disciple (§1NH II, 1-4). Apocryphe n'est pas à prendre dans le sens que nous lui donnons actuellement : le terme désigne ici, comme dans le titre du traité XIII de l'hermétisme un enseignement secret transmis à un disciple, qui sera chargé de le rapporter exclusivement à un groupe d'initiés. Comme l'explique Tardieu, la spécificité gnostique tient à l'expression «cachés dans le silence » : l'enseignement en question «n'appartient pas au logos démiurgique mais provient de l'Indicible qui est au-delà et tout entier silence $»^{20}$. Et même révélé par la parole, il ne doit pas être divulgué à tous.

18. Pour l'histoire du mot « gnostique », voir Dubois-Tardieu $1986: 21-37$.

19. Pour les sources directes et indirectes, voir Dubois-Tardieu 1986 et Dubois-Kuntzmann 1987.

20. Tardieu 1984, Codex de Berlin : 240. L'Apocryphon de Jean sera cité selon la traduction et la division en paragraphes de Tardieu. 
Le traité se conclut d'ailleurs par une recommandation : Je t'ai dit cela pour que tu le mettes par écrit et que tu le transmettes à tes compagnons spirituels secrètement (§78). Avant d'être soustrait au regard de Jean, le Sauveur prononce une malédiction contre toute mauvaise utilisation de l'ouvrage. Nous nous trouvons donc en présence d'un Jean ésotérique, à côté du selon Jean exotérique.

Le contenu de la révélation permet de comprendre la critique d'Irénée. En effet, la révélation présente une relecture des Ecritures dans le sens proprement gnostique. Le créateur de l'univers, pour les gnostiques, n'est pas le dieu parfait et ineffable. Le démiurge est l'œuvre de la Sagesse préexistante, Sophia, qui, en voulant agir seule, crée une déficience, soit directement, soit par un intermédiaire, l'ombre, comme dans l'Hypostase des Archontes: Et ce qu'elle (l'ombre, issue de Sophia) fit, fut une ouvre dans la matière, semblable à un avorton. Il reçut forme de l'ombre. Ce fut une bête arrogante, à la ressemblance d'un lion ${ }^{21}$. Cet être, que Sophia nomme Ialdabaoth, est le créateur du Judaïsme. Ialdabaoth reçoit de Sophia une partie de sa « lumière », ce qui introduit le thème de la chute de la lumière. Egaré par son orgueil, il se nomme lui-même dieu et affirme sa toute-puissance. L'Apocryphon de Jean illustre bien dans cet épisode comment se réalise le questionnement des Ecritures, avec, il faut le reconnaître, une logique certaine : Il (le démiurge) vit la création au-dessous de lui et la multitude des anges subalternes issus de lui, et il leur dit : "Je suis un dieu jaloux, en dehors de moi il n'y a personne ». Par là, il signifiait déjà aux anges subalternes qu'un autre dieu existe! S'il n'y en avait pas d'autre, de qui, en effet, pourrait-il bien être jaloux? (\$38). L'affirmation du dieu jaloux comme dieu exclusif se mue en manifestation de la stupidité du créateur ${ }^{22}$. Même si elle s'appuie sur une distorsion du sens que la langue grecque encourage, la méthode ne manque pas de subtilité, puisqu'il s'agit de tirer des Ecritures la preuve de l'interprétation donnée. S'il y a dans l'Ancien Testament un sens perdu, cette vérité se manifeste là où on l'attendrait le moins, dans la bouche du mauvais démiurge. On notera que cette leçon sur la méthode exégétique n'est pas présentée comme une spéculation humaine, mais provient de la bouche du Sauveur en personne, dans une révélation secrète. La vérité de l'interprétation est garantie par deux locuteurs : le créateur, même si c'est contraire à son intention, et Jésus qui livre le sens profond de la phrase.

Cette validation de la méthode par une telle autorité n'est certainement pas gratuite. Mais ce n'est pas la seule : la relecture de la Genèse permet aux gnostiques de fonder le thème du salut par la gnose sous forme de révélation ésotérique en le faisant remonter au plus loin, jusqu'aux ancêtres mythiques. Comme c'est souvent le cas, les différents traités divergent sur plusieurs points. Le schéma narratif qui suit ne tient pas compte de ces écarts. L'essentiel ici est de montrer comment un savoir caché, qui correspond à l'enseignement ésotérique des gnostiques, est une composante récurrente de l'histoire de l'humanité, et que les gnostiques historiques s'inscrivent dans une lignée traditionnelle. Leur doctrine ésotérique, et l'ésotérisme lui-même, se justifient parce qu'ils sont l'aboutissement d'un processus initié dès l'origine.

Le blasphème de Ialdabaoth, qui prétend être le seul dieu, provoque une réaction du dieu indicible, qui manifeste une ressemblance de lui. Les puissances mauvaises,

21. HA 94, 14-17, Barc 1980 : 67. Cf. ApJ, §§27-28.

22. Tardieu 1984, Codex de Berlin : 294. 
ou Archontes, décident de modeler un homme à la ressemblance de cette ressemblance, qu'ils installeront dans le paradis.

A côté de la figure de Ialdabaoth, les gnostiques posent celle de l'archonte Sabaoth, son fils ou son frère. Ce dernier incarne la fonction royale et la justice (c'est Yahveh), tandis que Ialdabaoth (Elohim) est la fonction démiurgique. Sabaoth, qui est le pôle surdéterminé de la paire, reçoit la portion de lumière tombée dans le monde. Lors de la création de l'homme, il lui insuffle une âme, et l'homme reçoit la lumière abandonnée par Sophia lors de la naissance du démiurge. De matériel, hylique, l'homme devient psychique. C'est cette lumière qui sera la source du salut.

C'est à ce stade que se définit le modèle mythique de la transmission du savoir ésotérique gnostique : Adam, pour accéder à l'état parfait de spirituel, ne peut le faire par spéculation philosophique. Il a besoin qu'une aide lui soit envoyée du divin pour l'instruire sur la vérité de ses origines. Cette aide, c'est Eve, la femme spirituelle : Et la femme spirituelle vint vers lui, elle parla avec lui, elle dit : "Lève-toi, Adam!" Et lorsqu'il la vit, il dit : "Tu es celle qui m'as donné la vie ; on t'appellera la mère des vivants » (HA 89, 11-15). L'Apocryphon de Jean définit la fonction de cette aide envoyée à Adam : Voilà celle qui vient en aide à la créature tout entière! Elle prend sur elle son tourment et la redresse en sa plénitude, elle l'instruit de la descente de (sa) déficience, et elle l'instruit de la voie de la remontée, (qui est) la voie par où elle est descendue. Et l'intelligence-lumière était cachée en Adam (non seulement) pour passer inaperçue des archontes, mais pour (permettre) à l'intelligence de redresser la déficience de la mère ( $\$ 54 \mathrm{~b}-\mathrm{c})$. Toujours d'après l'Apocryphon de Jean, les Archontes se rendent compte de la supériorité de l'homme et l'enferment dans la matière : Voilà notre lien! Voilà le tombeau du remodelage du corps! Voilà ce qu'ils ont fait endosser à l'homme, ces bandits : le lien de l'oubli! Et c'est ainsi que l'homme est devenu mortel (...) Quant à l'intelligence-lumière préexistante qui était en lui, (voilà celle) qui éveille sa pensée (\$55c).

Adam est enfermé dans le paradis, et dans la logique du gnosticisme, l'épisode biblique du serpent et de l'arbre de la connaissance du bien et du mal est lu d'une manière non-traditionnelle. Reprenons la narration de l'Hypostase des Archontes : suscitant le désir des Archontes, la femme spirituelle projette une ombre d'elle-même (la femme charnelle), que violentent les Archontes (cette union donnera naissance à Cain et Abel). Prenant la forme du serpent, elle incite la femme charnelle à goûter du fruit de l'arbre. De même, la femme charnelle en fait manger à Adam. Dans l'Apocryphon, l'arbre est l'intelligence-lumière, et Jésus révèle à Jean qu'il a lui même incité Adam à goûter du fruit défendu. Puisqu'il s'agit d'une forme dialoguée, l'auteur insère ici une question étonnée de Jean, qui souligne littérairement l'interprétation ésotérique du passage de la Genèse : Seigneur, n'est-ce pas plutôt le serpent qui apprit à manger à Adam? (\$59). A quoi le Sauveur sourit, et dans sa réponse, évite une assimilation explicite au serpent. L'homme sera chassé du paradis et maudit, après un dialogue où éclate l'ignorance du dieu biblique.

Si Cain et Abel sont issus d'une union violente et hybride des puissances mauvaises et de la femme charnelle, l'union d'Adam et de la femme spirituelle produit le prototype du gnostique, Seth, père de la race humaine. Seth reçoit comme son père une "aide ", Noréa, et les hommes se multiplient, jusqu'au moment où les Archontes méditent de les détruire. C'est le grand Archonte Sabaoth qui sauve Noé, mais le pacte ne permet pas à l'homme d'emporter avec lui son aide, sa contre-partie 
féminine. L'homme est alors coupé de la connaissance. Un ange, pourtant, instruit la femme et lui annonce que la vérité se manifestera à nouveau : les descendants de Seth, descendants d'Adam et de l'Eve spirituelle, qui recevront l'esprit deviendront des « parfaits » et recevront le salut. Ainsi, l'alliance du judaïsme, alliance entre Sabaoth et les Hébreux, inaugurée lors du déluge et renouvelée lors de l'épisode des Tables de la Loi, le prive de la bonne interprétation du livre qu'il écrit. Les élus, les gnostiques, recevant à nouveau la lumière, retrouvent le sens véridique. On le voit, il ne s'agit pas pour eux d'une nouvelle lecture, ni d'une meilleure lecture, mais de la seule lecture. Cette « lecture », comme l'a montré Barc, repose d'ailleurs sur une réécriture, qui implique l'addition ou la suppression de mots, la modification de l'ordre des mots ou des passages. Cette manipulation du texte se justifie par le mythe : ceux qui ont transmis le texte n'étaient pas détenteurs de sa signification et ont donc corrompu la vérité qu'il contient. En ce sens, les gnostiques ne maltraitent le texte que parce qu'ils sont dépositaires de son sens caché.

On l'a vu, la validation de cette lecture passe par différents moyens. L'un d'eux consiste à chercher dans les textes sacrés la preuve de leur interprétation. Le démiurge se proclamant jaloux leur en fournit une, qu'ils placent dans la bouche de Jésus ressuscité. La preuve est donnée par une autorité, qui la transmet à une autorité humaine : Jean dans l'Apocryphon, mais d'autres personnages, comme Thomas ou Marie-Madeleine, peuvent être dépositaires du savoir caché. D'autres techniques de validation coexistent, comme la découverte de stèles gravées (les stèles de Seth) et la transmission de leur contenu aux élus, qui retrouvent ainsi la connaissance d'un savoir perdu, remontant au prototype du gnostique. Le cadre littéraire des stèles gravées est de type égyptisant, ce qui n'a rien d'étonnant. La plupart de nos textes proviennent d'Egypte, et le lien entre la gnose et l'Egypte se fait aisément par le biais de l'hermétisme, dont les gnostiques utilisaient les textes comme ils utilisaient les philosophes grecs. On aurait tort d'imaginer une coupure entre les différentes cultures qui sont représentées en Egypte : les milieux philosophiques grecs, les milieux du judaïsme hellénisé, le christianisme naissant et les élites intellectuelles égyptiennes elles-mêmes. Il suffit de savoir qu'ils avaient une langue de culture commune, le grec, que des ouvrages circulaient, que les intellectuels de tout bord avaient la possibilité de se rencontrer, ne fût-ce qu'à Alexandrie, mais également dans d'autres centres de culture, en Egypte et en dehors de l'Egypte. La région thébaine, où fut trouvé l'essentiel des documents de première main (le corpus de Nag Hammadi), est un lieu important d'échanges culturels, comme l'ont remarquablement montré G. Fowden et J.-P. Mahé. La preuve d'un contact apparaît d'ailleurs dans un texte sacré égyptien du deuxième siècle de notre ère, au temple d'Esna, dans la région thébaine, qui attribue la naissance du désordre à l'activité démiurgique, un thème qui n'est pas égyptien, mais gnostique. Les auteurs gnostiques utilisent, à bon escient d'ailleurs, des éléments du judaïsme et de la cosmologie égyptienne, qui se retrouvent également dans les papyrus magiques grecs d'Egypte, par exemple sous la forme de noms magiques.

Cette circulation des savoirs cachés nous amène naturellement à nous poser la question du sens à donner à la notion de secret réservé à un cercle d'initiés. Si Adam, quand il désobéit au grand Archonte en mangeant du fruit de l'arbre, n'ose pas lui dire la vérité, les gnostiques, eux, n'ont plus cette timidité et l'existence de renégats ne suffit pas à expliquer la diffusion des idées. L'existence d'écrits exotériques témoigne d'un désir de convaincre et de faire de nouveaux adeptes. Mais les savoirs ésotériques 
de la gnose comme de l'hermétisme circulent en dehors des adeptes, à moins qu'il faille supposer, par exemple, qu'un prêtre égyptien de haut rang, un initié aux mystères égyptiens, ne soit également initié à d'autres mystères, même chrétiens. Ou faut-il suivre Umberto Eco, qui, comme on l'a dit plus haut, dans les Limites de l'interprétation, démonte le mécanisme du secret, qui renvoie toujours à un autre secret pour aboutir à un vide? Les gnostiques, pourtant, ne semblent pas se livrer à une sémiosis illimitée sur les textes. Il faut constater que les mêmes doctrines peuvent être présentes dans un ouvrage explicitement secret, comme l'Apocryphon de Jean, et dans une lettre adressée à quelqu'un qui souhaite s'informer sur les puissances, comme l'Hypostase des Archontes. Il arrive même qu'un écrit exotérique sous la forme d'un traité philosophique, comme Eugnoste, soit retravaillé pour lui donner l'habillement d'un écrit ésotérique de révélation, La Sagesse de Jésus-Christ. Le genre littéraire choisi par les auteurs ou les réviseurs des traités n'est certainement pas gratuit. Il serait pourtant abusif de considérer le genre de la révélation secrète comme un simple cadre littéraire au goût du jour, mais sans signification. On l'a vu, le thème du savoir caché, qui sert de fondement à l'anthropologie gnostique, justifie leur interprétation de la cosmologie. En cela, l'ésotérisme est inhérent à la gnose.

Michèle Broze, Chercheuse qualifiée au FNRS, Professeur à l'Université Libre de Bruxelles.

\section{RÉFÉRENCES BIBLIOGRAPHIQUES}

BARC B.

1980 L'Hypostase des Archontes. Traité gnostique sur l'origine de l'homme, du monde et des archontes, Bibliothèque copte de Nag Hammadi, section «Textes » 5. Québec : Presses Universitaires de Laval : $171 \mathrm{p}$.

BROZE M.

1995 Le politique et ses falsifications, Revue de Philosophie Ancienne XIII : 31-53.

BROZE M.

1996 Mythe et roman en Egypte ancienne. Les aventures d'Horus et Seth dans le Papyrus Chester Beatty I, OLA 76 : Leuven : Peeters : $304 \mathrm{p}$.

BROZE M.

1997 Temps réel, temps imaginaire et temps fictionnel dans la révélation hermétique, in WUNENBURGER J.J. (éd.), Figures du temps, Strasbourg : Presses Universitaires de Strasbourg : 109-120.

COULOUBARITSIS L.

1982 Le paradoxe du philosophe dans la République de Platon. Revue de Métaphysique et de Morale 87 : 60-81.

1962 L'authenticité de l'inspiration égyptienne dans le Corpus Hermeticum, RHR 161: 175-198. 
DESCLOS M.-L.

2000 Instituer la philosophie : le temps de la succession dans le Parménide de Platon, in DARBOPESCHANSKI C. (éd.), Constructions du temps dans le monde grec ancien, Paris : CNRSPhilosophie : 223-252.

DUBOIS J.-D., KUNTZMANN R

1987 Nag Hammadi. Evangile selon Thomas. Textes gnostiques aux origines du christianisme, Supplément au Cahier Evangile 58, Paris : Editions du Cerf : 178 p.

DUBOIS J.-D., TARDIEU M.

1986 Introduction à la littérature gnostique. I. Collections retrouvées avant 1945, Paris : Editions du Cerf : $152 \mathrm{p}$.

ECO U.

1992 Les limites de d'interprétation, Paris (Milan 1990) : Grasset : 406 p.

FESTUGIERE A.-J., NOCK A.D.

1983 (1954) Corpus hermeticum IV Fragments extraits de Stobée-Fragments divers, Paris : Les Belles Lettres : $150 \mathrm{p}$.

FESTUGIERE A.-J.

1944-1954 La révélation d'Hermès Trismégiste, Paris : J. Gabalda : 4 vols.

FOWDEN G.

1986 The Egyptian Hermes, Cambridge : Cambridge University Press : 244 p.

\section{KASAJIMA M.}

2001 Magie et théurgie dans le Corpus hermeticum XIII. Analyse de la structure et du cadre narratif, Mémoire présenté à 1'Université Libre de Bruxelles : 96 p.

LORY P.

1988 Hermès-Idris, prophète et sage dans la tradition islamique, in FAIVRE A. (éd.), Présence d'Hermès Trismégiste, Paris : Albin Michel : 100-109.

MAHÉ J.-P.

1978 Hermès en Haute Egypte, T. 1, Bibliothèque copte de Nag Hammadi, section « Textes » 3. Québec : Presses Universitaires de Laval : 171 p.

PUECH H.

1978 En quête de la gnose. I La gnose et le temps, Paris : Gallimard : 300 p.

TARDIEU M.

1984 Le codex de Berlin, Sources gnostiques et manichéennes, T. 1. Paris : Editions du Cerf : 518 p. 